\title{
Three Node Tandem Communication Network Model with Duane Arrival Process having Phase Type Service
}

\author{
Kalavala Asish Vardhan \\ Department of CS\&SE \\ Andhra University \\ Visakhapatnam \\ India
}

\author{
K. Srinivasa Rao \\ Department of Statistics \\ Andhra University \\ Visakhapatnam \\ India
}

\author{
P. Srinivasa Rao \\ Department of CS\&SE \\ Andhra University \\ Visakhapatnam \\ India
}

\begin{abstract}
This paper introduces a three node Tandem communication network model in which the arrival process follows a Duane process such that the inter arrival times follow Weibull distribution. In this model it is assumed that intermediator departures after transmission from first and second nodes are allowed it is further assumed that the inter transmission times in each node follow Poisson process and the transmission strategy is Dynamic bandwidth allocation. The network behavior is analyzed by deriving expressions for average number of packets in each Queue. The mean delays in transmission, the throughput of the nodes, the Utilization of the transmitters and the variability of the content of buffers. Through Sensitivity analysis it is observed that the arrival process has significant influence on network performance measures. The strategy of allowing inter mediatory departures reduce conjunction in buffers and mean delay of transmission of packets. This model also includes some of the earlier models as particular cases for specific limiting for specific values of the parameter. This model is useful for design and monitoring self-Similarity networks.
\end{abstract}

\section{Keywords}

Duane Arrival process, Communication network model, Sensitivity analysis, DBA

\section{INTRODUCTION}

William Cock and Charles Wheatstone [1] have pioneered the mathematical theory of communication. Thereafter in 1948 a tremendous revolution in communication network modeling is brought by Barden and Barttain. Later AT\&T Bell labs, USA network group at MIT, IEEE communication society and other reputed organizations have put considerable efforts for invoking and designing efficient communication systems. (IEEE Communication Society, 2002). Conducting laboratory experiments under variable load conditions is highly complex and time consuming. Hence for efficient design and evaluation of communication networks, the network models are developed and analyzed with various assumptions on constituent processes of the model like arrival process, service process, flow control mechanisms, allocations, routing, etc. To improve Quality of Service, packet switching gives better utilization over circuit or message switching and yields relatively shorter delay in statistical multiplexing in communication network can reduce the delay in packet switching. Many of communication networks which support teleprocessing applications are mixed with dynamic engineering skills and statistical multiplexing [2], [3], [4]. To reduce the congestion in buffers the dynamic bandwidth allocation is evolved as an alternative and efficient control strategy over bit dropping or flow control strategies [5], [7], $[8,9]$. In DBA, a large portion of the unutilized bandwidth is utilized by changing the transmission rate of packets depending on the content of the buffer connected to it. Much work has been reported recently regarding communication network model with dynamic bandwidth [10]. In all these papers, the authors assumed that the arrival rate of packets is constant and follows a Poisson process.

But in many communication systems the assumption regarding Poisson process are seldom satisfied due to the time dependent nature of arrivals. Therefore, many phenomenon encountered in communication systems which reveal time dependent behavior of arrival process due to the factors like work load fluctuations, initiating, failures, congestion and flow control, overload peaks, reconfigurations, adaptive routing and others. So to have an efficient performance evaluation entities problem of communication systems like adaptive isolated routing and load balancing, evaluating alternative buffer of changed sharing schemes and effects and to study the effects of flow and control strategies it is needed to develop communication network models with time dependent arrivals. In addition to this [10] have showed that the packet arrival process of heavy tail distribution inter arrivals form a non homogeneous process and the mean packet arrival rate is not constant but it is time varying. This is also supported by the studies [18], who demonstrated the TCP connection arrival process is bursty and time dependent. Recently, much emphasis is given for modeling the arrival process of the communication networks using, Nonhomogeneous Poisson Process with the assumption that the arrival rate is linearly dependent on time [9] [10], [11, 12]. In all these papers they assumed that the burstness of the traffic is either linearly increasing or decreasing depending on time, but in LAN, WAN, and MAN the traffic structure is quite different from the, arrival rates being linearly dependent function of time. This is also supported by [18] who have, modeled the packet arrivals as Log Normal distributions, since, the associated network traffic inter arrivals are distributed with heavy tail distributions. Feldmann [18] demonstrated that the Transport Control Protocol (TCP) connection arrival bursty and are characterized by heavy tail distributions such as Weibull distributions. Fisher et al [19], has analyzed a singer server queuing model with Weibull inter arrivals using simulations studied. Araik Tamazian and Mikhail Bogachev [17] have used Weibull distributions for inter arrival times in analyzing the performance of World Wide Web (WWW) servers. In these studies, they consider that the server is single and the queues are independent. But in LAN, WAN and MAN the output of one queue is an input to the other queue, and queues are not independent. No work has been reported in literature regarding Tandem communication network models with Weibull inter arrival times which model the LAN, WAN, MAN and other selfsimilarity network traffic with time dependent bursts, more close to the reality. This motivated to develop and analyze a two node tandem communication network model with Weibull inter arrival times having dynamic band width 
allocation with modified phase type transmission. The Weibull distribution includes exponential distribution and several other skewed distributions as particular cases. Using the differential calculus, the explicit expressions for the network performance parameters such as mean number of packets in each buffer, mean delay in transmission, the throughput of the nodes, and the variability of the buffer content are derived. Through numerical analysis the performance of the communication network is evaluated with respect to the changes in input parameters. A comparative study of the proposed model with that of exponential inter arrival times is also carried.

\section{COMMUNICATION NETWORK MODEL AND TRANSIENT SOLUTION}

In this section, a communication network model having three nodes in tandem is studied. The arrivals to the buffer connected at node one is assumed to follow a Duane process with mean arrival rate as a function of time $t$. It is of the form $\lambda(t)=a b t b-1$. The transmission process from node one to node two follows a Poisson process with parameter $\mu 1$. It is also assumed that the packets arrive to the second buffer directly from outside of the network in a Poisson process with mean arrival rate $\epsilon$. After getting transmitted from node one the packets are forwarded to the second buffer for transmission with probability $\theta$ or get terminated with probability $\pi$ i.e., the packets arrived at second buffer contains the packets received from first node and directly from outside. After getting transmitted from second node the packet are forwarded to the third buffer for transmission with probability $\pi$ or get terminated with probability $(1-\pi)$. The transmission process of node two and three also follow Poisson process with parameters $\mu 2$ and $\mu 3$ respectively. The transmission rate of each packet is adjusted just before transmission depending on the content of the buffer connected to the transmitter. The packets are transmitted through the transmitters by the first in first out discipline. The schematic diagram representing the communication network model is shown in Figure 1.

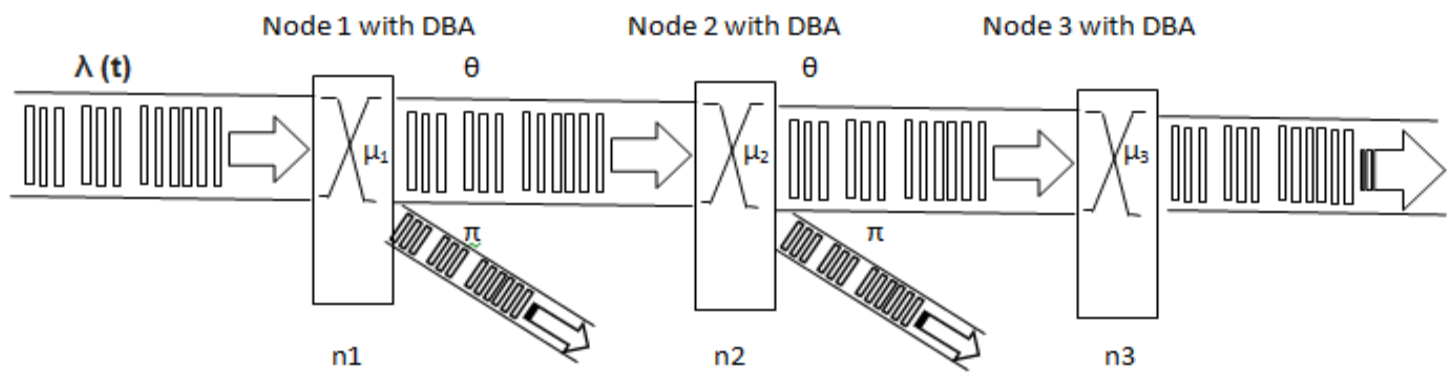

Figure 1: Schematic diagram of the CNM model with three stage arrival

Let $P n_{1}, n_{2}, n_{3}(t)$ be the probability that there are $n_{1}$ packets in the first buffer and $n_{2}$ packets in the second buffer and $n_{3}$ packets in the third buffer at the time t. with this structure, the difference -differential equations of the communication network are :

\section{PERFORMANCE MEASURES OF THE NETWORK}

The mean number of packets in the first buffer is

$$
L_{1}(t)=E\left[N_{1}\right]=\sum_{n_{1}=0}^{\infty} n_{1} P_{n_{1}}(t)=\left[a b\left[\left[e^{-\mu_{1} t} \int_{0}^{t} e^{-\mu_{1} v} v^{b-1} d v-\frac{e^{-\mu t}}{\mu_{1}}\right]\right]\right]
$$

The utilization of the first buffer is

$$
\begin{aligned}
& U_{1}(t)=1-P_{0}(t) \\
& =\left[1-\exp \left[-a b\left[e^{-\mu_{1} t} \int_{0}^{t} e^{-\mu_{1} v} v^{b-1} d v-\frac{e^{-\mu_{1} t}}{\mu_{1}}\right]\right]\right]
\end{aligned}
$$

For computing the variability of the number of packets in first buffer is

$$
E\left(N_{1}^{2}-N_{1}\right)=
$$

Therefore, the variance of the number of packets in the first buffer is

$\operatorname{Var}\left(N_{1}\right)=a b\left[e^{-\mu_{1} t} \int_{0}^{t} e^{-\mu_{1} v} v^{b-1} d v-\frac{e^{-\mu_{1} t}}{\mu_{1}}\right]$
In this section, the performance measures of the communication network under transient conditions are derived and analyzed. Expanding $P\left(s_{1}, s_{2}, s_{3} ; t\right)$ given equation (16) and collecting the constant terms, the probability that the network is empty achieved as follows,

The throughput of the first transmitter is

$T h p_{1}(t)=\mu_{1}\left(1-P_{0}(t)\right)=\mu_{1}\left[1-\exp \left[-a b\left[e^{-\mu_{t} t} \int_{0}^{t} e^{-\mu_{1} v} v^{b-1} d v-\frac{e^{-\mu t_{t}}}{\mu_{1}}\right]\right]\right]$

The mean delay in the first buffer size is

$W_{1}(t)=\frac{L_{1}(t)}{T h p_{1}(t)}$

Similarly, by substituting $s_{1}=1$, and $s_{3}=1, s_{1}=1$, and $s_{2}=1$, the probability generating function of the second buffer and third buffer size distributions are achieved and values are tabulated as follows,

\section{PERFORMANCE EVALUATION OF THE NETWORK}

In this section, the performance of the developed communication network is discussed through a numerical illustration. Different values of the parameters are considered for bandwidth allocation and arrival of packets. After interacting with the technical staff an internet providing station, is considered the packet arrival rate parameter "a" varies from $11 \times 10^{4}$ packets/sec to $14 \times 10^{4}$ packets/sec, and ' $b$ ' varies from 1.25 to 2.5 with an average packet size of 52 bytes. After transmitting from node 1 , the forward 
transmission rate ' $\mu_{1}$ ' varies from $11 \times 10^{4}$ packets/sec to $14 \times 10^{4}$ packets/sec. The rate of transmission rate ' $\mu_{2}$ ' varies from $26 \times 10^{4}$ packets $/ \mathrm{sec}$ to $29 \times 10^{4}$ packets $/ \mathrm{sec}$. The rate of transmission rate ' $\mu_{3}$ ' varies from $28 \times 10^{4}$ packets/sec to $31 \times 10^{4}$ packets $/ \mathrm{sec}$. In all these nodes, dynamic bandwidth allocation strategy is considered i.e., the transmission rate of each packet depends on the number of packets in the buffer connected to it at that instant

Since performance characteristics of communication network are highly sensitive with respect to time, the transient behavior of the model is studied through computing the performance measures with the set of values for the model parameters as $\mathrm{t}=0.8,0.9,1.0,1.1$ seconds, $\mathrm{a}=11,12,13,14$ (with multiplication to $10 \times 10^{4}$ packets/sec) $b=1.25,1.5,2,2.5$ $\mu_{1}=11,12,13,14\left(10 \times 10^{4}\right.$ packets/sec $), \mu_{2}=26,27,28,29$

(10x104packets/sec), $\mu 3=28,29,30,31(10 \times 104$ packets/sec), $\pi$ $=0.4,0.3,0.2,0.1, \theta=0.6,0.7,0.8,0.9$.

The probability of the network emptiness and buffer emptiness are computed for different values of $t, a, b, \mu_{1}, \mu_{2}$, $\mu_{3}, \pi, \theta$ and presented in Table 1 . The probability of network emptiness and different buffers emptiness with various parameters are represented graphically in Figure 2. From the Table 1 it is observed that the probability of emptiness in the entire communication network and in three buffers are highly sensitive with respect to changes in time. As time ' $\mathrm{t}$ ' varies from 0.8 seconds to 1.1 seconds, the probability of emptiness in the network reduces from 0.2261 to 0.0496 when other parameters are fixed at $(10,3,10,25,30,0.5,0.5)$ for $\left(a, b, \mu_{1}, \mu_{2}\right.$, $\left.\mu_{3}, \pi, \theta\right)$. Similarly, the probability for emptiness in three buffers vary from 0.2234 to $0.0483,0.7649$ to 0.5713 and 0.9036 to 0.8042 for node 1 , node 2 and node 3 respectively. The decrease in the node 1 is more rapid when compared to node 2 , node 3 . The influence of mean arrival rate of packets on the system emptiness is also studied. As the arrival rate parameters 'a' varies from $11 \times 10^{4}$ packets/sec to $14 \times 10^{4}$ packets/sec, the probability of emptiness of the network decreases from 0.1946 to 0.1242 when other parameters are fixed at $(0.8,3,10,25,30,0.5,0.5)$ for $\left(t, b, \mu_{1}, \mu_{2}, \mu_{3}, \pi, \theta\right)$. The same phenomenon is observed with respect to three nodes. This decrease is more in first node and moderate in second and third nodes.

Table 1: Values of network and buffer emptiness probability and utilization of the communication network with DBA*=seconds, $\$=$ multiples of 1000 packets/seconds.

\begin{tabular}{|c|c|c|c|c|c|c|c|c|c|c|c|c|c|c|}
\hline$t^{*}$ & a\$ & b & $\mu_{1} \$$ & $\boldsymbol{\mu}_{2} \$$ & $\mu_{3} \$$ & $\pi$ & $\theta$ & $\mathbf{P}_{0,0,0}(t)$ & $P_{0}, ., .(t)$ & $P_{., 0, .,}(t)$ & $P ., ., 0(t)$ & $\mathbf{U}_{1}(\mathbf{t})$ & $\mathbf{U}_{2}(\mathbf{t})$ & $\mathbf{U}_{\mathbf{3}}(\mathbf{t})$ \\
\hline 0.8 & 10 & 3 & 10 & 25 & 30 & 0.5 & 0.5 & 0.2261 & 0.2234 & 0.7649 & 0.9036 & 0.7766 & 0.2351 & 0.0962 \\
\hline 0.9 & 10 & 3 & 10 & 25 & 30 & 0.5 & 0.5 & 0.1447 & 0.1423 & 0.7023 & 0.8735 & 0.8577 & 0.2977 & 0.1264 \\
\hline 1.0 & 10 & 3 & 10 & 25 & 30 & 0.5 & 0.5 & 0.0872 & 0.0854 & 0.6372 & 0.8403 & 0.9146 & 0.3628 & 0.1597 \\
\hline 1.1 & 10 & 3 & 10 & 25 & 30 & 0.5 & 0.5 & 0.0496 & 0.0483 & 0.5713 & 0.8042 & 0.9517 & 0.4287 & 0.1957 \\
\hline 0.8 & 11 & 3 & 10 & 25 & 30 & 0.5 & 0.5 & 0.1946 & 0.1923 & 0.7447 & 0.8945 & 0.8077 & 0.2553 & 0.1053 \\
\hline 0.8 & 12 & 3 & 10 & 25 & 30 & 0.5 & 0.5 & 0.1676 & 0.1655 & 0.725 & 0.8855 & 0.8345 & 0.275 & 0.1143 \\
\hline 0.8 & 13 & 3 & 10 & 25 & 30 & 0.5 & 0.5 & 0.1442 & 0.1425 & 0.7058 & 0.8765 & 0.8575 & 0.2942 & 0.1232 \\
\hline 0.8 & 14 & 3 & 10 & 25 & 30 & 0.5 & 0.5 & 0.1242 & 0.1226 & 0.6872 & 0.8677 & 0.8774 & 0.3128 & 0.1320 \\
\hline 0.8 & 10 & 1.25 & 10 & 25 & 30 & 0.5 & 0.5 & 0.3281 & 0.3199 & 0.799 & 0.9119 & 0.6801 & 0.201 & 0.0881 \\
\hline 0.8 & 10 & 1.5 & 10 & 25 & 30 & 0.5 & 0.5 & 0.293 & 0.2864 & 0.7846 & 0.9062 & 0.7136 & 0.2154 & 0.0937 \\
\hline 0.8 & 10 & 2 & 10 & 25 & 30 & 0.5 & 0.5 & 0.2513 & 0.2467 & 0.7681 & 0.9008 & 0.7533 & 0.2319 & 0.0991 \\
\hline 0.8 & 10 & 2.5 & 10 & 25 & 30 & 0.5 & 0.5 & 0.2322 & 0.2287 & 0.7629 & 0.9006 & 0.7713 & 0.2371 & 0.0992 \\
\hline 0.8 & 10 & 3 & 11 & 25 & 30 & 0.5 & 0.5 & 0.2514 & 0.2482 & 0.7601 & 0.9014 & 0.7518 & 0.2399 & 0.0985 \\
\hline 0.8 & 10 & 3 & 12 & 25 & 30 & 0.5 & 0.5 & 0.2757 & 0.2722 & 0.756 & 0.8995 & 0.7278 & 0.244 & 0.1004 \\
\hline 0.8 & 10 & 3 & 13 & 25 & 30 & 0.5 & 0.5 & 0.2991 & 0.2952 & 0.7524 & 0.8979 & 0.7048 & 0.2476 & 0.1021 \\
\hline 0.8 & 10 & 3 & 14 & 25 & 30 & 0.5 & 0.5 & 0.3214 & 0.3172 & 0.7494 & 0.8965 & 0.6828 & 0.2506 & 0.1035 \\
\hline 0.8 & 10 & 3 & 10 & 26 & 30 & 0.5 & 0.5 & 0.2261 & 0.2234 & 0.772 & 0.9032 & 0.7766 & 0.228 & 0.0966 \\
\hline 0.8 & 10 & 3 & 10 & 27 & 30 & 0.5 & 0.5 & 0.226 & 0.2234 & 0.7787 & 0.9028 & 0.7766 & 0.2213 & 0.097 \\
\hline 0.8 & 10 & 3 & 10 & 28 & 30 & 0.5 & 0.5 & 0.2259 & 0.2234 & 0.7849 & 0.9025 & 0.7766 & 0.2151 & 0.0974 \\
\hline 0.8 & 10 & 3 & 10 & 29 & 30 & 0.5 & 0.5 & 0.2259 & 0.2234 & 0.7909 & 0.9021 & 0.7766 & 0.2091 & 0.0977 \\
\hline 0.8 & 10 & 3 & 10 & 25 & 28 & 0.5 & 0.5 & 0.2262 & 0.2234 & 0.7649 & 0.8978 & 0.7766 & 0.2351 & 0.102 \\
\hline 0.8 & 10 & 3 & 10 & 25 & 29 & 0.5 & 0.5 & 0.2261 & 0.2234 & 0.7649 & 0.9036 & 0.7766 & 0.2351 & 0.0962 \\
\hline 0.8 & 10 & 3 & 10 & 25 & 30 & 0.5 & 0.5 & 0.2261 & 0.2234 & 0.7649 & 0.9063 & 0.7766 & 0.2351 & 0.0935 \\
\hline 0.8 & 10 & 3 & 10 & 25 & 31 & 0.5 & 0.5 & 0.2261 & 0.2234 & 0.7649 & 0.9063 & 0.7766 & 0.2351 & 0.0935 \\
\hline 0.8 & 10 & 3 & 10 & 25 & 30 & 0.4 & 0.6 & 0.2257 & 0.2234 & 0.807 & 0.9073 & 0.7766 & 0.193 & 0.0925 \\
\hline 0.8 & 10 & 3 & 10 & 25 & 30 & 0.3 & 0.7 & 0.2252 & 0.2234 & 0.8515 & 0.9184 & 0.7766 & 0.1485 & 0.0815 \\
\hline 0.8 & 10 & 3 & 10 & 25 & 30 & 0.2 & 0.8 & 0.2246 & 0.2234 & 0.8984 & 0.9372 & 0.7766 & 0.1016 & 0.0627 \\
\hline 0.8 & 10 & 3 & 10 & 25 & 30 & 0.1 & 0.9 & 0.224 & 0.2234 & 0.9478 & 0.9642 & 0.7766 & 0.0522 & 0.0358 \\
\hline
\end{tabular}




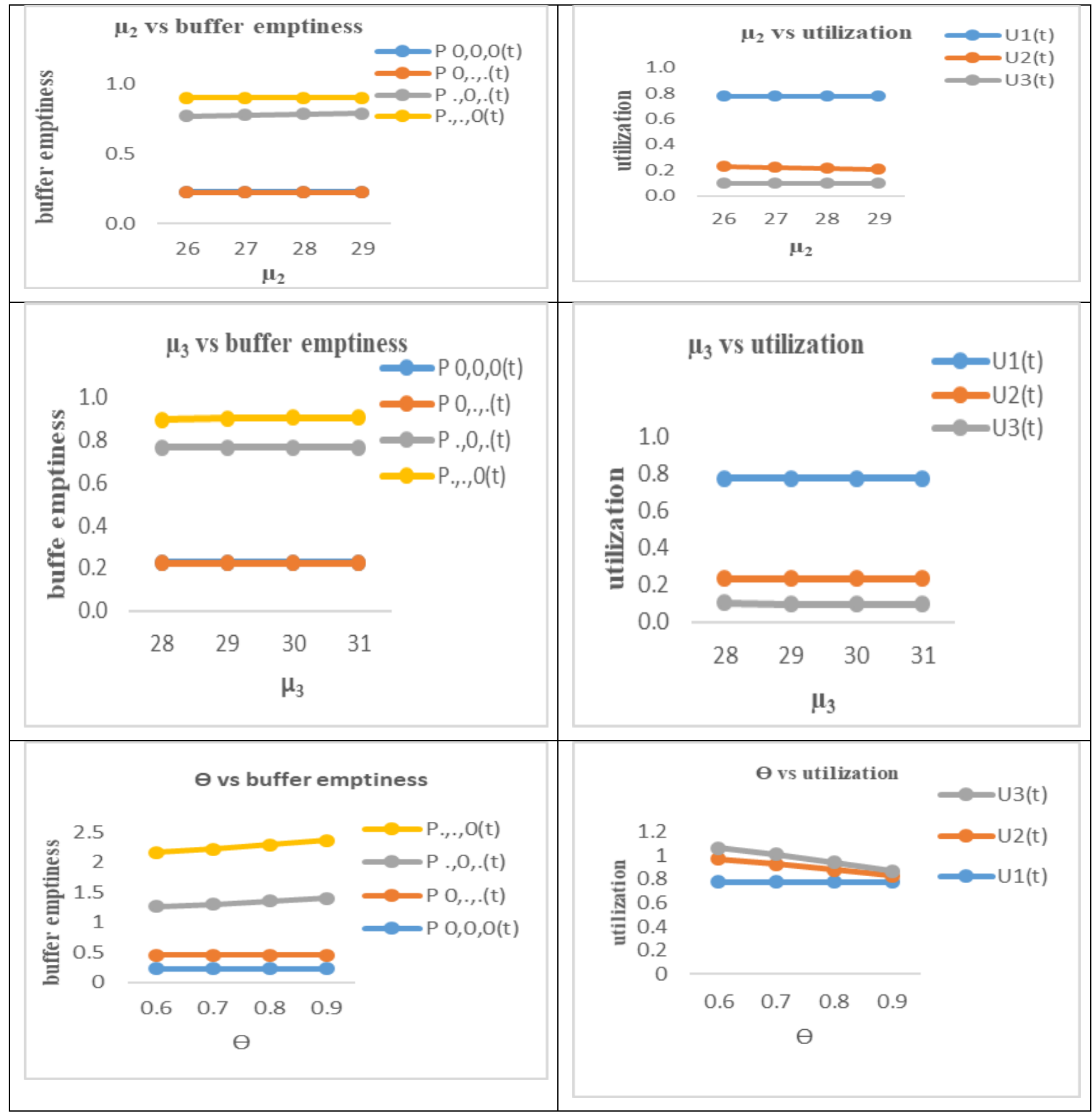

When the arrival rate parameter ' $b$ ' varies from 1.25 to 2.5 , the probability of emptiness of the network decreases from 0.3281 to 0.2322 when the other parameters remain fixed. The same phenomenon is observed for three nodes. The decrease is faster in node 1 and moderate in the next two nodes.

When the transmission rate ' $\mu_{1}$ ' of node 1 varies from $11 \times 10^{4}$ packets/sec to $14 \times 10^{4}$ packets/sec the probability of emptiness in the network increase from 0.2514 to 0.3214 when the other parameters remain fixed. Similarly, the transmission rate ' $\mu_{2}$ ' of node2 varies from $26 \times 10^{4}$ packets/sec to $29 \times 10^{4}$ packets/sec. The probability of emptiness of the network increases from 0.2261 to 0.2259 when the other parameters remain fixed. Similarly, the transmission rate ' $\mu_{3}$ ' of node 3 varies from $28 \times 10^{4}$ packets/sec to $31 \times 10^{4}$ packets/sec. The probability of emptiness in the network increases from 0.2262 to 0.2261 when the other parameters remain fixed. The same phenomenon is observed with respect to the emptiness of the three buffers.

When arrival rate Parameter ' $\theta$ ' increases from 0.6 to 0.9 , the probability of emptiness in the network decreases from 0.2257 to 0.224 when other parameter is remaining fixed. The utilization of transmitters of node 1 , node 2 and node 3 for different parameters are computed and presented in Table 1 and the relationship with performance measures is shown in Figure 2. As time't' and arrival rate parameter 'a' increase the utilization of transmitters are increasing for fixed values of the other parameters. It is also observing that the as the arrival rate parameter ' $a$ ' increases the utilization of transmitters in three nodes are increasing for fixed values of the other parameters. As the transmission rate ' $\mu_{1}$ ' increases, the utilization of the first node decreases. Similarly, as the 
transmission rate ' $\mu_{2}$ ' increases the utilization of the second node decreases when the other parameters remain fixed.

Similarly, as the transmission rate ' $\mu_{3}{ }^{\prime}$ increases the utilization of in third node decreases when the other parameters remain fixed.
The mean number of packets in the buffer and in the network, mean delays in transmission of both nodes are computed for different values are $t, a, b, \mu_{1}, \mu_{2}, \mu_{3}, \pi, \theta$ and presented at Table 2. The relationship between the parameters and the performance measures are shown in the Figure 3.

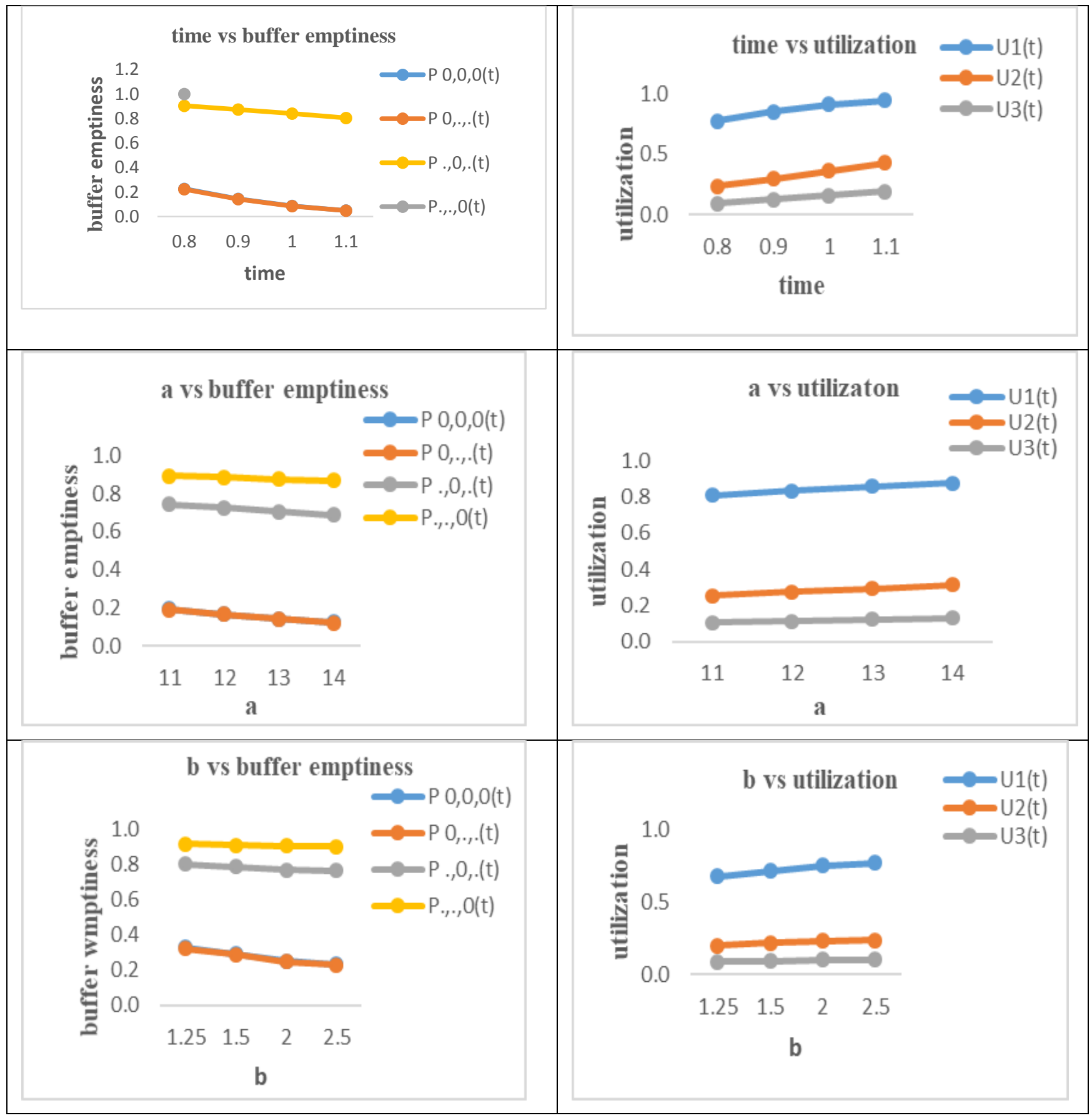


Table 2: Values of mean number of packets and mean delay of the communication network with DBA *=seconds, $\$=$ multiples of 1000 packets/seconds

\begin{tabular}{|c|c|c|c|c|c|c|c|c|c|c|c|c|c|c|}
\hline $\mathbf{t}^{*}$ & a\$ & b & $\mu 1 \$$ & $\mu 2 \$$ & $\mu 3 \$$ & $\pi$ & $\theta$ & $L_{1}(t)$ & $\mathbf{L}_{2}(\mathbf{t})$ & $\mathbf{L}_{3}(\mathbf{t})$ & $L_{n}(t)$ & $W_{1}(t)$ & $\mathbf{W}_{2}(\mathbf{t})$ & $\mathbf{W}_{\mathbf{3}}(\mathbf{t})$ \\
\hline 0.8 & 10 & 3 & 10 & 25 & 30 & 0.5 & 0.5 & 1.4990 & 0.2680 & 0.1011 & 1.8681 & 0.1930 & 0.0456 & 0.0350 \\
\hline 0.9 & 10 & 3 & 10 & 25 & 30 & 0.5 & 0.5 & 1.9496 & 0.3534 & 0.1351 & 2.4381 & 0.2273 & 0.0475 & 0.0356 \\
\hline 1.0 & 10 & 3 & 10 & 25 & 30 & 0.5 & 0.5 & 2.4599 & 0.4507 & 0.1740 & 3.0845 & 0.2690 & 0.0497 & 0.0363 \\
\hline 1.1 & 10 & 3 & 10 & 25 & 30 & 0.5 & 0.5 & 3.0299 & 0.5599 & 0.2178 & 3.8077 & 0.3184 & 0.0522 & 0.0371 \\
\hline 0.8 & 11 & 3 & 10 & 25 & 30 & 0.5 & 0.5 & 1.6489 & 0.2948 & 0.1113 & 2.0549 & 0.2041 & 0.0462 & 0.0352 \\
\hline 0.8 & 12 & 3 & 10 & 25 & 30 & 0.5 & 0.5 & 1.7988 & 0.3216 & 0.1214 & 2.2417 & 0.2156 & 0.0468 & 0.0354 \\
\hline 0.8 & 13 & 3 & 10 & 25 & 30 & 0.5 & 0.5 & 1.9487 & 0.3484 & 0.1315 & 2.4285 & 0.2272 & 0.0474 & 0.0356 \\
\hline 0.8 & 14 & 3 & 10 & 25 & 30 & 0.5 & 0.5 & 2.0986 & 0.3752 & 0.1416 & 2.6153 & 0.2392 & 0.0480 & 0.0357 \\
\hline 0.8 & 10 & 1.25 & 10 & 25 & 30 & 0.5 & 0.5 & 1.1399 & 0.2244 & 0.0922 & 1.4565 & 0.1676 & 0.0447 & 0.0349 \\
\hline 0.8 & 10 & 1.5 & 10 & 25 & 30 & 0.5 & 0.5 & 1.2504 & 0.2425 & 0.0983 & 1.5913 & 0.1752 & 0.045 & 0.035 \\
\hline 0.8 & 10 & 2 & 10 & 25 & 30 & 0.5 & 0.5 & 1.3994 & 0.2638 & 0.1043 & 1.7675 & 0.1858 & 0.0455 & 0.0351 \\
\hline 0.8 & 10 & 2.5 & 10 & 25 & 30 & 0.5 & 0.5 & 1.4753 & 0.2707 & 0.1045 & 1.8505 & 0.1913 & 0.0457 & 0.0351 \\
\hline 0.8 & 10 & 3 & 11 & 25 & 30 & 0.5 & 0.5 & 1.3934 & 0.2744 & 0.1037 & 1.7715 & 0.1685 & 0.0457 & 0.0351 \\
\hline 0.8 & 10 & 3 & 12 & 25 & 30 & 0.5 & 0.5 & 1.3012 & 0.2798 & 0.1058 & 1.6868 & 0.149 & 0.0459 & 0.0351 \\
\hline 0.8 & 10 & 3 & 13 & 25 & 30 & 0.5 & 0.5 & 1.2201 & 0.2844 & 0.1077 & 1.6122 & 0.1332 & 0.046 & 0.0352 \\
\hline 0.8 & 10 & 3 & 14 & 25 & 30 & 0.5 & 0.5 & 1.1484 & 0.2885 & 0.1093 & 1.5461 & 0.1201 & 0.046 & 0.0352 \\
\hline 0.8 & 10 & 3 & 10 & 26 & 30 & 0.5 & 0.5 & 1.499 & 0.2588 & 0.1016 & 1.8594 & 0.193 & 0.0437 & 0.0351 \\
\hline 0.8 & 10 & 3 & 10 & 27 & 30 & 0.5 & 0.5 & 1.499 & 0.2502 & 0.102 & 1.8512 & 0.193 & 0.0419 & 0.0351 \\
\hline 0.8 & 10 & 3 & 10 & 28 & 30 & 0.5 & 0.5 & 1.499 & 0.2422 & 0.1024 & 1.8435 & 0.193 & 0.0402 & 0.0351 \\
\hline 0.8 & 10 & 3 & 10 & 29 & 30 & 0.5 & 0.5 & 1.499 & 0.2346 & 0.1028 & 1.8364 & 0.193 & 0.0387 & 0.0351 \\
\hline 0.8 & 10 & 3 & 10 & 25 & 28 & 0.5 & 0.5 & 1.499 & 0.268 & 0.1076 & 1.8746 & 0.193 & 0.0456 & 0.0377 \\
\hline 0.8 & 10 & 3 & 10 & 25 & 29 & 0.5 & 0.5 & 1.499 & 0.268 & 0.1011 & 1.8681 & 0.193 & 0.0456 & 0.035 \\
\hline 0.8 & 10 & 3 & 10 & 25 & 30 & 0.5 & 0.5 & 1.499 & 0.268 & 0.0982 & 1.8651 & 0.193 & 0.0456 & 0.0339 \\
\hline 0.8 & 10 & 3 & 10 & 25 & 31 & 0.5 & 0.5 & 1.499 & 0.268 & 0.0982 & 1.8651 & 0.193 & 0.0456 & 0.0339 \\
\hline 0.8 & 10 & 3 & 10 & 25 & 30 & 0.4 & 0.6 & 1.499 & 0.2144 & 0.0971 & 1.8105 & 0.193 & 0.0444 & 0.035 \\
\hline 0.8 & 10 & 3 & 10 & 25 & 30 & 0.3 & 0.7 & 1.499 & 0.1608 & 0.085 & 1.7447 & 0.193 & 0.0433 & 0.0348 \\
\hline 0.8 & 10 & 3 & 10 & 25 & 30 & 0.2 & 0.8 & 1.499 & 0.1072 & 0.0647 & 1.6709 & 0.193 & 0.0422 & 0.0344 \\
\hline 0.8 & 10 & 3 & 10 & 25 & 30 & 0.1 & 0.9 & 1.499 & 0.0536 & 0.0364 & 1.589 & 0.193 & 0.0411 & 0.0339 \\
\hline
\end{tabular}

It is observed that when time $\mathrm{t}=0.8$ seconds the mean number of packets in the first buffer is 14990 packets after one seconds it is rapidly increases to 24599 packets, and at 1.1 seconds it becomes 30299 packets and there after its stabilizes. The mean number of packets in the second and third buffers in the network are increasing from 2680 packets to 5599 packets, from 1011 to 2178 packets when other parameters are fixed when the arrival rate parameter 'a' varies from $11 \times 10^{4}$ packets/sec to $14 \times 10^{4}$ packets/sec, the mean number of packets in the first, second and third buffers in the network are increasing from 16489 to 20986 packets, 2948 packets to 3752 packets 1113 packets to 1416 packets respectively. The parameter ' $b$ ' varies from 1.25 to 2.5 the mean number of packets in the first, second and third buffer in the network are increasing from 11399 packets to 14753 packets, 2244 packets to 2707 packets, 0922 packets to 1402 packets respectively, when other parameters remain fixed.

The transmission parameter ' $\mu_{1}$ ' varies from $11 \mathrm{x} 10^{4}$ packets/sec to $14 \times 10^{4}$ packets/sec, the mean number of packets in the first, second and third buffers are increasing from 13934 packets to 11484 packets, from

2744 packets to 2885 packets, from 1037 packets to 1093 packets respectively, when other parameters remain fixed.

Similarly, the transmission rate ' $\mu_{2}{ }^{\prime}$ varies from $26 \times 10^{4}$ packets $/ \mathrm{sec}$ to $29 \times 10^{4}$ packets $/ \mathrm{sec}$, the mean number of packets in the first buffer remains at 14990 packets and the mean number of packets in the second and third buffers are 
increasing from 2588 packets to 2346 packets, and 1016 packets to 1028 packets respectively when other parameters are remaining fixed. Similarly, the transmission parameter ' $\mu_{3}$ ' varies from $28 \times 10^{4}$ packets $/ \mathrm{sec}$ to $31 \times 10^{4}$ packets $/ \mathrm{sec}$, the mean number of packets in the first and second buffers remain constant at 14990 packets and 268 packets respectively. and third buffer decreases from 1076 to 0982 when other parameters remain fixe

It is observed that as time ' $\mathrm{t}$ ' and the arrival parameters ' $a$ ' $b$ ' are increasing the mean delay in buffers are increasing for fixed values of the other parameters. It

is also observed that as parameter ' $\theta$ ' varies the mean delay in buffers are decreasing for fixed values of the other parameters.

The parameters $\pi$ and, $\theta$ varies the mean delay in first buffer remain constant and in the second and third buffers its values are decreasing for fixed values of the other parameters. The variance of the number of packets in each buffer and in the network, throughput of each node are computed for different values of $t, a, b, \mu_{1}, \mu_{2}, \mu_{3} \pi, \theta$ are presented in Table 3 . It is observed as time ' $t$ ' increases, the throughput of first, second and third nodes are increasing from 77664 packets to 95168 packets, 58769 to 107184 packets and 28859 packets to 58725 packets respectively.

As the arrival parameter 'a' varies from $11 \times 10^{4}$ packets/sec to $14 \times 10^{4}$ packets $/ \mathrm{sec}$, the throughput of the first, second and third nodes are increasing from 80773 packets to 87737 packets, from 63825 to 78207 packets and from 31587 packets to 39609 packets. As the arrival parameter 'b' varies from $1.25 \times 10^{4}$ packets/sec to $2.5 \times 10^{4}$ packets $/ \mathrm{sec}$, the throughput of the first, second and third nodes are increasing from 68015 packets to 77129 packets, from 50250 to 59284 packets and from 26419 packets to 29773packets.

When the transmission parameter " $\mu_{1}$ " varies from $11 \times 10^{4}$ packets $/ \mathrm{sec}$ to $14 \times 10^{4}$ packets $/ \mathrm{sec}$, the throughput of the first, second and third nodes are increasing from 82695 packets to 95598 packets, from 59986 to 62650 packets and from 29547 packets to 31054 packets when other parameters remain fixed. The transmission parameter " $\boldsymbol{\mu}_{2}$ " varies from $26 \times 10^{4}$ packets/sec to $29 \times 10^{4}$ packets/sec, the throughput of the first node remain constant at 77664 packets, for the second and third nodes its values are increasing from packets to 59282packets top 60645 packets, and from 2893 packets to 29306 packets respectively when other parameters are remaining fixed. Similarly, the transmission parameter " $\mu_{3}$ " varies from $28 \times 10^{4}$ packets/sec to $31 \times 10^{4}$ packets $/ \mathrm{sec}$, the throughput of the first and second nodes remain constant at 77664 packets and 58769 packets respectively and for third node it increases from 28565 packets to 28993 packets, respectively when other parameters are fixed. The graphical representation for the table 2 and table 3 are also calculated in thesimilarfashion.

Table 3: Effect of Various Parameters on Throughput and Variance under Transient State for Communication Network Model *=seconds, \$= multiples of 1000 packets/seconds

\begin{tabular}{|c|c|c|c|c|c|c|c|c|c|c|c|c|c|}
\hline $\mathbf{t}^{*}$ & a\$ & b & $\mu 1 \$$ & $\mu 2 \$$ & $\mu 3 \$$ & $\pi$ & $\boldsymbol{\theta}$ & $\operatorname{Var}\left(\mathrm{N}_{1}\right)$ & $\operatorname{Var}\left(\mathbf{N}_{2}\right)$ & $\operatorname{Var}\left(\mathbf{N}_{\mathbf{3}}\right)$ & $\operatorname{Thp}_{1}(t)$ & $\operatorname{Thp}_{2}(t)$ & $\operatorname{Thp}_{3}(t)$ \\
\hline 0.8 & 10 & 3 & 10 & 25 & 30 & 0.5 & 0.5 & 1.4990 & 0.2680 & 0.1011 & 7.7664 & 5.8769 & 2.8859 \\
\hline 0.9 & 10 & 3 & 10 & 25 & 30 & 0.5 & 0.5 & 1.9496 & 0.3534 & 0.1351 & 8.5767 & 7.4425 & 3.7914 \\
\hline 1.0 & 10 & 3 & 10 & 25 & 30 & 0.5 & 0.5 & 2.4599 & 0.4507 & 0.1740 & 9.1455 & 9.0700 & 4.7909 \\
\hline 1.1 & 10 & 3 & 10 & 25 & 30 & 0.5 & 0.5 & 3.0299 & 0.5599 & 0.2178 & 9.5168 & 10.7184 & 5.8725 \\
\hline 0.8 & 11 & 3 & 10 & 25 & 30 & 0.5 & 0.5 & 1.6489 & 0.2948 & 0.1113 & 8.0773 & 6.3825 & 3.1587 \\
\hline 0.8 & 12 & 3 & 10 & 25 & 30 & 0.5 & 0.5 & 1.7988 & 0.3216 & 0.1214 & 8.3450 & 6.8748 & 3.4288 \\
\hline 0.8 & 13 & 3 & 10 & 25 & 30 & 0.5 & 0.5 & 1.9487 & 0.3484 & 0.1315 & 8.5754 & 7.3541 & 3.6962 \\
\hline 0.8 & 14 & 3 & 10 & 25 & 30 & 0.5 & 0.5 & 2.0986 & 0.3752 & 0.1416 & 8.7737 & 7.8207 & 3.9609 \\
\hline 0.8 & 10 & 1.25 & 10 & 25 & 30 & 0.5 & 0.5 & 1.1399 & 0.2244 & 0.0922 & 6.8015 & 5.025 & 2.6419 \\
\hline 0.8 & 10 & 1.5 & 10 & 25 & 30 & 0.5 & 0.5 & 1.2504 & 0.2425 & 0.0983 & 7.1362 & 5.3839 & 2.8096 \\
\hline 0.8 & 10 & 2 & 10 & 25 & 30 & 0.5 & 0.5 & 1.3994 & 0.2638 & 0.1043 & 7.5325 & 5.7968 & 2.9719 \\
\hline 0.8 & 10 & 2.5 & 10 & 25 & 30 & 0.5 & 0.5 & 1.4753 & 0.2707 & 0.1045 & 7.7129 & 5.9284 & 2.9773 \\
\hline 0.8 & 10 & 3 & 11 & 25 & 30 & 0.5 & 0.5 & 1.3934 & 0.2744 & 0.1037 & 8.2695 & 5.9986 & 2.9547 \\
\hline 0.8 & 10 & 3 & 12 & 25 & 30 & 0.5 & 0.5 & 1.3012 & 0.2798 & 0.1058 & 8.7336 & 6.1011 & 3.0126 \\
\hline 0.8 & 10 & 3 & 13 & 25 & 30 & 0.5 & 0.5 & 1.2201 & 0.2844 & 0.1077 & 9.1625 & 6.1889 & 3.0623 \\
\hline 0.8 & 10 & 3 & 14 & 25 & 30 & 0.5 & 0.5 & 1.1484 & 0.2885 & 0.1093 & 9.5598 & 6.265 & 3.1054 \\
\hline 0.8 & 10 & 3 & 10 & 26 & 30 & 0.5 & 0.5 & 1.499 & 0.2588 & 0.1016 & 7.7664 & 5.9282 & 2.8983 \\
\hline 0.8 & 10 & 3 & 10 & 27 & 30 & 0.5 & 0.5 & 1.499 & 0.2502 & 0.102 & 7.7664 & 5.9764 & 2.9098 \\
\hline 0.8 & 10 & 3 & 10 & 28 & 30 & 0.5 & 0.5 & 1.499 & 0.2422 & 0.1024 & 7.7664 & 6.0218 & 2.9206 \\
\hline 0.8 & 10 & 3 & 10 & 29 & 30 & 0.5 & 0.5 & 1.499 & 0.2346 & 0.1028 & 7.7664 & 6.0645 & 2.9306 \\
\hline
\end{tabular}




\begin{tabular}{|c|c|c|c|c|c|c|c|c|c|c|c|c|c|}
\hline 0.8 & 10 & 3 & 10 & 25 & $\mathbf{2 8}$ & 0.5 & 0.5 & 1.499 & 0.268 & 0.1076 & 7.7664 & 5.8769 & 2.8565 \\
\hline 0.8 & 10 & 3 & 10 & 25 & $\mathbf{2 9}$ & 0.5 & 0.5 & 1.499 & 0.268 & 0.1011 & 7.7664 & 5.8769 & 2.8859 \\
\hline 0.8 & 10 & 3 & 10 & 25 & $\mathbf{3 0}$ & 0.5 & 0.5 & 1.499 & 0.268 & 0.0982 & 7.7664 & 5.8769 & 2.8993 \\
\hline 0.8 & 10 & 3 & 10 & 25 & $\mathbf{3 1}$ & 0.5 & 0.5 & 1.499 & 0.268 & 0.0982 & 7.7664 & 5.8769 & 2.8993 \\
\hline 0.8 & 10 & 3 & 10 & 25 & 30 & $\mathbf{0 . 4}$ & $\mathbf{0 . 6}$ & 1.499 & 0.2144 & 0.0971 & 7.7664 & 4.824 & 2.7759 \\
\hline 0.8 & 10 & 3 & 10 & 25 & 30 & $\mathbf{0 . 3}$ & $\mathbf{0 . 7}$ & 1.499 & 0.1608 & 0.085 & 7.7664 & 3.7132 & 2.4435 \\
\hline 0.8 & 10 & 3 & 10 & 25 & 30 & $\mathbf{0 . 2}$ & $\mathbf{0 . 8}$ & 1.499 & 0.1072 & 0.0647 & 7.7664 & 2.5412 & 1.8804 \\
\hline 0.8 & 10 & 3 & 10 & 25 & 30 & $\mathbf{0 . 1}$ & $\mathbf{0 . 9}$ & 1.499 & 0.0536 & 0.0364 & 7.7664 & 1.3046 & 1.0727 \\
\hline
\end{tabular}

If the variance of the number of packets in each buffer increases, then the burstness of the buffer will be high. Hence, the parameters are to be adjusted in such a way that the variance of the content of each buffer is become small. The coefficients of variation of the number of packets in each buffer is computed. To understand the consistency of the traffic flow. Its help us to control the smooth flow of packets in buffers. From this analysis it is observe that the dynamic bandwidth allocation strategy has significant influence on all

\section{COMPARATIVE STUDY}

The comparative study between the proposed communication network model with that of homogenous Poisson arrivals is presented in this section, the computed performance measure of both models are presented in the Table 5 for different values of $\mathrm{t}=0.8,0.9,1.0,1.1$. performance measures of the network. It is further observed that the performance measures are highly sensitive towards smaller values of time. Hence, it is optimal to consider dynamic bandwidth allocation under and Duane arrival process and evaluate the performance under transient conditions. It is also to be observed that the congestion in buffer and delays in transmission got reduced to a minimum level by adopting dynamic bandwidth allocation. This phenomenon has a vital bearing on quantity of transmission.

As time $t$ increases, the percentage variation of performance measures between the models are increasing. For the proposed models with non-homogenous Poisson arrivals having dynamic bandwidth allocation has more utilization compared to that of the model with Poisson arrivals under dynamic bandwidth allocation. From this analysis it is observed that the assumption of non-homogenous arrivals has a significant influence on all the performance measures of the network.

Table 4: Comparative study of models with homogeneous and Non-homogeneous Duane Arrivals

\begin{tabular}{|c|c|c|c|c|c|}
\hline Time & Parameters & Poisson & Proposed model & Difference & Variance \\
\hline 0.8 & $\mathrm{~L}_{1}(\mathrm{t})$ & 0.9993 & 1.499 & 0.4997 & 33.33555704 \\
\cline { 2 - 6 } & $\mathrm{L}_{2}(\mathrm{t})$ & 0.1998 & 0.268 & 0.0682 & 25.44776119 \\
\cline { 2 - 6 } & $\mathrm{L}_{3}(\mathrm{t})$ & 0.0832 & 0.1011 & 0.0179 & 17.70524233 \\
\cline { 2 - 6 } & $\mathrm{U}_{1}(\mathrm{t})$ & 0.6319 & 0.7766 & 0.1447 & 18.63250064 \\
\cline { 2 - 6 } & $\mathrm{U}_{2}(\mathrm{t})$ & 0.1811 & 0.2351 & 0.054 & 22.96894938 \\
\cline { 2 - 6 } & $\mathrm{U}_{3}(\mathrm{t})$ & 0.0798 & 0.0962 & 0.0164 & 17.04781705 \\
\cline { 2 - 6 } & $\mathrm{Thp}_{1}(\mathrm{t})$ & 6.3187 & 7.7664 & 1.4477 & 18.64055418 \\
\cline { 2 - 6 } & $\mathrm{Thp}_{2}(\mathrm{t}$ & 4.5272 & 5.8769 & 1.3497 & 22.96618966 \\
\cline { 2 - 6 } & $\mathrm{Thp}_{3}(\mathrm{t})$ & 2.3948 & 2.8859 & 0.4911 & 17.01722166 \\
\cline { 2 - 6 } & $\mathrm{W}_{1}(\mathrm{t})$ & 0.1582 & 0.193 & 0.0348 & 18.03108808 \\
\cline { 2 - 6 } & $\mathrm{W}_{2}(\mathrm{t})$ & 0.0441 & 0.0456 & 0.0015 & 3.289473684 \\
\cline { 2 - 6 } & $\mathrm{W}_{3}(\mathrm{t})$ & 0.0347 & 0.035 & 0.0003 & 0.857142857 \\
\hline \multirow{4}{*}{0.9} & $\mathrm{~L}_{1}(\mathrm{t})$ & 0.9998 & 1.9496 & 0.9498 & 48.71769 \\
\cline { 2 - 6 } & $\mathrm{L}_{2}(\mathrm{t})$ & 0.1999 & 0.3534 & 0.1535 & 43.4352 \\
\cline { 2 - 6 } & $\mathrm{L}_{3}(\mathrm{t})$ & 0.0833 & 0.1351 & 0.0518 & 38.34197 \\
\cline { 2 - 6 } & $\mathrm{U}_{1}(\mathrm{t})$ & 0.632 & 0.8577 & 0.2257 & 26.31456 \\
\cline { 2 - 6 } & $\mathrm{U}_{2}(\mathrm{t})$ & 0.1812 & 0.2977 & 0.1165 & 39.13336 \\
\cline { 2 - 6 } & $\mathrm{U}_{3}(\mathrm{t})$ & 0.0799 & 0.1264 & 0.0465 & 36.78797 \\
\cline { 2 - 6 } & $\mathrm{Th}_{1}(\mathrm{t})$ & 6.3203 & 8.5767 & 2.2564 & 26.30849 \\
\hline
\end{tabular}




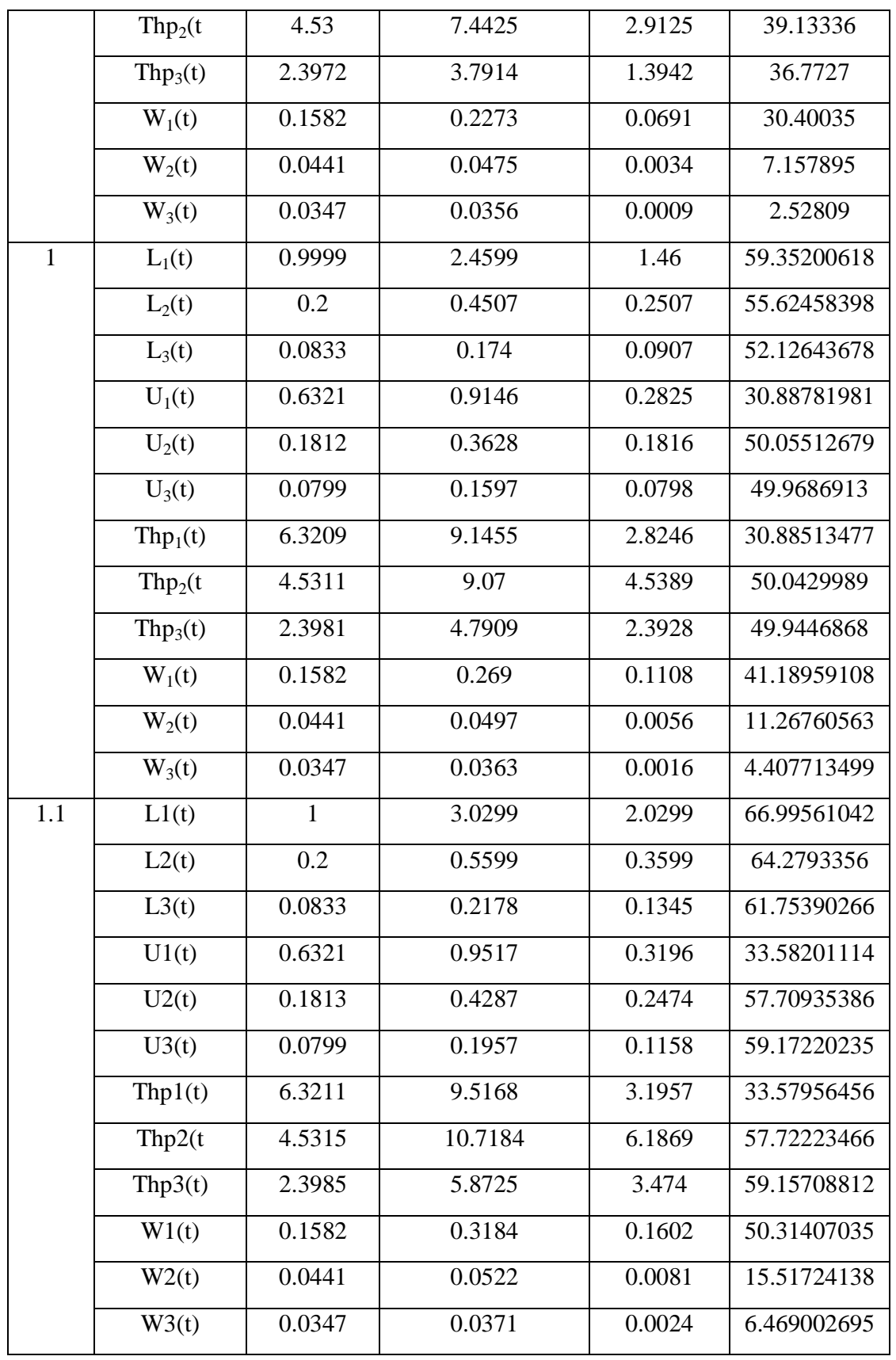

\section{CONCLUSION}

In this paper, a new and novel three nodes tandem communication network model with Duane arrival process having dynamic bandwidth allocation with phase type transmission was introduced. The arrival of packets in a network traffic is characterized through Weibull inter arrival time distribution which better represents the self-similarity network traffic having time dependent burstness. The Duane process is a generalization of Poisson process. Here it is, assumed that the three nodes are connected in tandem and the transmission processes follow Poisson. The transmission rate at every instant of packet transmission is adjusted depending on the content of the buffer connected to it. The explicit expressions for the performance measures of the communication network such as mean content of the buffers mean delays in transmission, the throughput of the nodes, utilization of the buffers are derived. The sensitivity of the model revealed that the inter arrival rate parameters has significant influence on predicting the performance measures of the network more accurately. A comparative study of the proposed network with that of inter exponential transmission times shown that the proposed model outperforms the existing communication network model in scheduling self-similarity networks such as LAN, WAN and MAN. It is, also possible to derive the optimal operating policies of the networks with suitable cost consideration which will be taken elsewhere. 


\section{REFERENCES}

[1] William cook and Charles Wheatstone (1939), IEEE communication society (2002).

[2] Gaujal. B, Hyon.E (2002) optimal routing policies in deterministic queues in tandem, proceedings of south international work shop on discrete event systems, pp:251-257.

[3] Parthasarathy.P.R. R and Selvaraju.N (2001) Transient analysis of a queue where potential customers are discouraged by queue length. Mathematical problem in engineering, Vol. 7, pp:433-454

[4] Srinivasa Rao., Vasantha. M.R, Vijaya Kumar. C.V.R.S (2002) on an independent communication network, opsearch vol.37, No.2, pp: 134-143.

[5] Sriram. K (1993) methodologies for bandwidth allocation transmission scheduling and congestion avoidance in broadband ATM networks, Computer network, ISDN system, J.26, pp:43-59

[6] Suresh Varma. P, Srinivasa Rao K, P. Srinivasa Rao (2007) a communication with load dependent transmission, International journal of mathematical sciences, vol.12, No.2, pp:1-15

[7] Padmavathi, G., Srinivasa Rao, K and Reddy, K.V.V.S (2009), "Performance Evaluation of parallel and series communication network with dynamic band width allocation CIIT" International, Journal of Networking and Communication, Vol.1, No.7, pp:410-42.

[8] Nageswara Rao, K., Srinivasa Rao, K. and Srinivasa Rao, P. (2010), "A tandem communication network with dynamic band width allocation and modified phase type transmission having bulk arrivals, International Journal of Computer Sciences, Issues, Vol. 7, No.2, pp:18-26.

[9] Kuda nageswarararao, K. Srinivasa Rao and P. Srinivasa Rao (2011) - "transient analysis of a tandem communication network with dynamic bandwidth allocation having two stage direct bulk arrivals, international journal of computer applications, vol.13, no.7, pp.14-22. Rakesh Singhai, Shiva Dutt Joshi and Rajendra K. P. Bhatt, (2007), "A Novel Discrete Distribution and Process to Model Self-SimilarTraffic, " 9 th IEEE international conference on telecommunications - Con TEL 2007, Zagreb, Croatia, pp 167-1

[10] Kuda nageswarararao, K. Srinivasa Rao and P. Srinivasa Rao (2011) - "transient analysis of a tandem communication network with dynamic bandwidth allocation having two stage direct bulk arrivals, international journal of computer applications, vol.13, no.7, pp.14-22.

[11] Kuda Nageswarararao Rao, K. Srinivasa Rao and P. Srinivasa Rao (2011) - performance evaluation of a communication network with dynamic bandwidth allocation and bulk arrivals, performance evaluation, vol.16, no.8.

[12] Padmavathi, G., Srinivasa Rao, K and Reddy, K.V.V.S (2009), "Performance Evaluation of parallel and series communication network with dynamic band width allocation CIIT" International, Journal of Networking and Communication, Vol.1, No.7, pp:410-42.

[13] P.Abry, R. Baraniuk, P. Flandrin, R. Riedi, D. Veitch, (2002), "Multi-scale Nature of Network Traffic," IEEE Signal Processing Magazine, pp. 28-27.

[14] Cappe O, E. Moulines, J. C. Pesquet, A. Petropulu, X. Yang (2002), "Long-Range Dependence and Heavy-Tail Modeling for Teletraffic Data," IEEE signal Processing Magazine, pp.14-27.

[15] W.Leland, M. Taqqu, W. Willinger, and D. Wilson,(1994) "On the self-similar nature of Ethernet traffic(extended version)," IEEE/ACM Trans. Networking, vol. 2, pp

[16] Crovella (1997) ME and Bestarros A (1997) selfsimilarity in worldwide traffic, evidence and possible cases, IEEE / ACM transaction networking, vol.5, No.6, pp:835-846

[17] A. Feldmann, (2000), "characteristics of TCP connection Arrivals" chapter 15, Self-Similar Network traffic and Performance evolution, edited by K.Park, W. Willinger, John Wiley\& Sons Inc.

[18] M. J. Fisher, D. Gross, D. Masi and J. F. Shortle, (2001), "Analyzing the Waiting Time Process in Internet Queuing Systems with the Transform Approximation Method," The Telecommunications Review,12, pp 21-32 